\title{
Biallelic mutations in carbamoyl phosphate synthetase 1 induced hyperammonemia in a neonate: A case report
}

\author{
JUN XU, AIMIN ZHANG and FURONG HUANG \\ Department of Neonatology, Children's Medical Center, Hunan Provincial People's Hospital and \\ The First Affiliated Hospital of Hunan Normal University, Changsha, Hunan 410005, P.R. China
}

Received October 18, 2018; Accepted September 20, 2019

DOI: $10.3892 /$ etm.2020.8717

\begin{abstract}
The aim of the present report was to describe the clinical presentation, diagnosis, and treatment of a case of carbamoyl phosphate synthetase 1 (CPS1) deficiency in a neonate, specifically, a 3 day-old female who visited Hunan Provincial People's Hospital due to anorexia and lethargy for 1 day. Physical and laboratory examination, and MRI were undertaken. Whole exome sequencing (WES) was applied for molecular etiology identification. Sanger sequencing was utilized to validate the variants detected by WES. Structural modeling was conducted for pathogenic analysis. Clinical examination revealed increased intracranial pressure, hyperammonemia, reduced citrulline, and increased glutamic acid levels. WES identified compound heterozygosity of c.713G $>C$, p.Arg238Pro and c.2339G >A, p.Arg780His in CPS1 (NCBI reference sequence, NM_001875.4) as candidate pathogenic variants. Sanger sequencing validated these variants. Structural modeling further confirmed the pathogenesis of these mutations. In conclusion, CPS1 deficiency in neonates is a serious condition that may be misdiagnosed due to severe infection. WES can be a helpful tool in facilitating the diagnosis of this disease.
\end{abstract}

\section{Introduction}

Urea cycle disorders (UCDs) are a set of hereditary metabolic disorders caused by congenital enzymatic defects that are characterized by high levels of ammonia in the blood (1). The urea cycle eliminates blood ammonia by transforming toxic ammonia into non-toxic urea, which can then be excreted in urine (2). Defects in any of the enzymes associated with urea

Correspondence to: Professor Aimin Zhang, Department of Neonatology, Children's Medical Center, Hunan Provincial People's Hospital and The First Affiliated Hospital of Hunan Normal University, 61 Liberation West Road, Changsha, Hunan 410005, P.R. China

E-mail: 24235759@qq.com

Key words: carbamoyl phosphate synthetase 1, hyperammonemia, urea cycle disorders metabolism [namely carbamoyl-phosphate synthetase (CPS1), ornithine carbamoyltransferase, argininosuccinate synthetase, argininosuccinate lyase, arginase and $\mathrm{N}$-acetyl glutamate synthase] may lead to the occurrence of UCDs (3). CPS1 deficiency can present as life-threatening hyperammonemia, a $\mathrm{UCD}$ and a rare autosomal recessive disorder of ureagenesis (4).

The human CPS1 gene is located at chromosome 2q34-35 and contains 38 exons and 4,500 coding nucleotides (5-8). CPS1 deficiency affects neonatal development and the severity of the symptoms is largely parallel to the degree of enzymatic defects, with complete enzyme deficiency being the most severe (4). Neonatal onset CPS deficiency leads to a poor prognosis, and patients who accept treatment with long-term protein restriction and ammonia medication may experience severe sequelae (9). The current study presents the clinical performance, diagnosis, and treatment of a case of CPS1 deficiency in a neonate and aims to increase understanding of the causes and symptoms of CPS1 deficiency and present new options for genetic screening.

\section{Case report}

Ethics. The present study was approved by the Ethics Committee of Hunan Provincial People's Hospital. This study complied with the World Medical Association Declaration of Helsinki (10) regarding the ethical conduct of research involving human subjects. Informed consent was obtained from the parents of the patient.

Patient. A 3 day-old female was admitted to the Department of Neonatology, Hunan Provincial People's Hospital, China in June 2016 due to anorexia and lethargy for 1 day. The patient was born to healthy non-consanguineous Chinese parents following full-term gestation, with a birth weight of $3.7 \mathrm{~kg}$. Her mother was gravida 3, para 2. No abnormality was noted within $48 \mathrm{~h}$ of the birth. However, her parents noted drowsiness and poor responsiveness, without obvious cause, after $72 \mathrm{~h}$. This condition gradually worsened. As the patient had a healthy 7 year old sister, abnormality based on family medical history was discarded as a reason for the condition.

Patient examination. Upon admission, routine examinations, including physical examination, urinalysis, blood gas analysis, and blood examination, were performed as described 
previously $(11,12)$. In addition, laboratory tests for C-reactive protein, pro-calcitonin, toxoplasma, rubella, cytomegalovirus and herpes simplex viruses, respiratory viruses (influenza virus, respiratory syncytial virus and adenovirus), biochemistry, routine cerebrospinal fluid analysis, blood ammonia, blood glucose, lactic acid, liver function and renal function were performed $(12,13)$. Bacterial cultures of blood, urine, and cerebrospinal fluid samples were conducted. Electrolytes, myocardial enzyme and magnetic resonance imaging (MRI) examinations were performed (11). In addition, lumbar puncture manometry was conducted. Liquid chromatography with tandem mass spectrophotometry was performed in order to analyze compounds in the serum of the patient. Venous blood was collected and dropped on to a filter paper of $\sim 10 \mathrm{~mm}$ in diameter and cooled at room temperature. Amino, organic and fatty acid detection was then performed as described previously (14).

Whole exome sequencing (WES). WES was performed according to a previously reported method (11). Briefly, genomic DNA was extracted from $2 \mathrm{ml}$ of peripheral blood from each family member using a BloodGen Midi kit (Beijing CWBio Co., Ltd.). The DNA was then sheared, hybridized, and enriched following the manufacturer's protocol. Size, distribution and concentration of DNA was assessed using an Agilent Bioanalyzer 2100 (Agilent Technologies Inc.). The libraries were sequenced on an Illumina Hiseq2500 platform (Illumina, Inc.).

Bioinformatics analysis. Raw sequencing files were processed using BclToFastq software (version 2; Illumina, Inc.). Variations were only included in the study where the quality score was $\geq 20$. The National Center for Biotechnology Information human reference genome (HG-19) was employed for the alignment of the sequencing reads. Single nucleotide polymorphisms and insertion-deletions in the sequences were analyzed with Genome Analysis Toolkit software (version.3.7; Broad Institute). Four online programs, including SIFT-SNP prediction (http://snpeff.sourceforge.net/SnpSift.html), PolyPhen2 (v 2, 2013; http://genetics.bwh.harvard.edu/pph2/) (15), Protein Variation Effect Analyzer (PROVEAN; 2012, http://provean. jcvi.org/index.php), and MutationTaster (v2, 2014, http://www. mutationtaster.org), were used to predict the pathogenicity of the variants. In the SIFT-SNP software, a score $>0.05$ indicated that a change of amino acids could be tolerated (16), while a score $<0.05$ indicated that a change of amino acids could not be tolerated, a lower score indicated a potentially poor tolerance of an amino acid change. In the PolyPhen2 software, a score close to 0.00 meant that the change of amino acids could be tolerated, while a score close to 1.00 meant that the change of amino acids could not be tolerated, and a higher score indicated that great damage could potentially be caused by amino acid change. In the PROVEAN software, if the score was equal to or below a predefined threshold (e.g. -2.5) (17), the protein variant was predicted to have a 'deleterious' effect, while if the PROVEAN score was above the threshold, the variant was predicted to have a 'neutral' effect (18). Mutation Taster scores were obtained using the tools available at http://www.mutationtaster.org/info/PhyloP_PhastCons_Test. html and previously described protocols (19).
Sanger sequencing. Sanger sequencing was conducted to validate the variants identified in the proband as described previously (12). The primers used were 5'-TCCCTTTAAGGA ATGGTTAGTCAAG-3' and 5'-GTCATGATAAGGAAC CATGAGTTG-3' for c.713G>C (p.Arg238Pro); and 5'-TCC TGTGACCTGTGCCTCTATAC-3', and 5'-ACACTGTCC ATGTGTTGATGGTATC-3' for c.2339G >A (p.Arg780His), which harbor 540 and 517-bp products, respectively. Genomic DNA, extracted from a blood sample from each of the three family members, was extracted with Qiagen FlexiGene DNA kit (cat. no. 512206; Qiagen $\mathrm{GmbH}$ ) according the protocol provided by the manufacturer. The DNA polymerase used was EasyTaq ${ }^{\circledR}$ (TransGen Biotech Co., Ltd.). The reaction conditions were as follows: Initial denaturation at $95^{\circ} \mathrm{C}$ for $5 \mathrm{~min} ; 32$ cycles of denaturation at $95^{\circ} \mathrm{C}$ for $45 \mathrm{sec}$, annealing at $56^{\circ} \mathrm{C}$ for $45 \mathrm{sec}$ and extension at $72^{\circ} \mathrm{C}$ for $1 \mathrm{~min}$ and a final extension at $72^{\circ} \mathrm{C}$ for $5 \mathrm{~min}$. The polymerase chain reaction products were sequenced using an ABI 3730XL (Applied Biosystems; Thermo Fisher Scientific, Inc.) and were analyzed with DNASTAR 5.0 software (DNASTAR, Inc.).

Structural modeling. The reported mutational sites of CPS1 were analyzed using the online server of the Human Gene Mutation Database (HGMD®; www.hgmd.cf.ac.uk; HGMD professional 2016.1 release). The crystal structure of CPS1 (apo form) was downloaded from the Protein Data Bank (PDB) database (https://www.ncbi.nlm.nih.gov/Structure/pdb/5DOU, released in Sep, 2015) in PDB format (20). PyMol (version 1.8.0.7; www.pymol.org) was used for structure displaying and structural figure preparation.

Clinical characteristics. Physical examination results were as follows: Temperature $38^{\circ} \mathrm{C}$; Pulse, 168 times/min; Respiratory Rate, 57 time/min; blood pressure, 60/32 mmHg; weight, $3.29 \mathrm{~kg}$; and head circumference, $34 \mathrm{~cm}$. The proband was in a light coma and her anterior fontanelle was $2.5 \times 2.5 \mathrm{~cm}$ in size, with high tension. Her pupils had equal diameters and were insensitive to papillary light reflex. The liver was palpable $2 \mathrm{~cm}$ below the right subcostal arch. The patient exhibited limb hypertonia.

Auxiliary examination results. For accurate diagnosis, an auxiliary examination was conducted following admission. Normal range values are based on accepted values in Hunan Provincial People's Hospital. A blood test revealed white blood cells, $26.48 \times 10^{9}$ cells $/ 1$ (normal range, $5-25 \times 10^{9}$ cells $/ 1$ ), neutrophil percentage, $61.5 \%$ (normal range, $50-70 \%$ ); hemoglobin, $183 \mathrm{~g} / 1$ (normal range, 145-220 g/l); platelet count, $341 \times 10^{9}$ cells $/ 1$ (normal range, $150-600 \times 10^{9}$ cells/1), C-reactive protein (CRP), $<3 \mathrm{mg} / \mathrm{dl}$ (normal, $\leq 3 \mathrm{mg} / \mathrm{dl}$ ); blood glucose, $4.8 \mathrm{mg} / \mathrm{dl}$ (normal range, 2.6-7.0 mg/dl); and blood ammonia, $109.7 \mu \mathrm{mol} / 1$ (normal, $\leq 40 \mu \mathrm{mol} / \mathrm{l}$ ). The index of inflammation was generally normal and the clinical data did not support infection. Liver and kidney function together with electrolyte levels were normal. Blood gas results demonstrated $\mathrm{pH}, 7.35$ (normal range, 7.30-7.40); partial pressure $\mathrm{CO}_{2}, 41 \mathrm{mmHg}$ (normal range, 35-45 $\mathrm{mmHg}$ ); partial pressure $\mathrm{O}_{2}, 78 \mathrm{mmHg}$ (normal range, 60-80 mmHg); Base Excess (BE), -1 mmol/1 (normal, $-6 \mathrm{mmol} /$ ); and lactic acid, $1.3 \mathrm{mmol} / 1$ (normal, $\leq 2 \mathrm{mmol} / \mathrm{l}$ ). Blood tandem mass spectrometry revealed that 
A

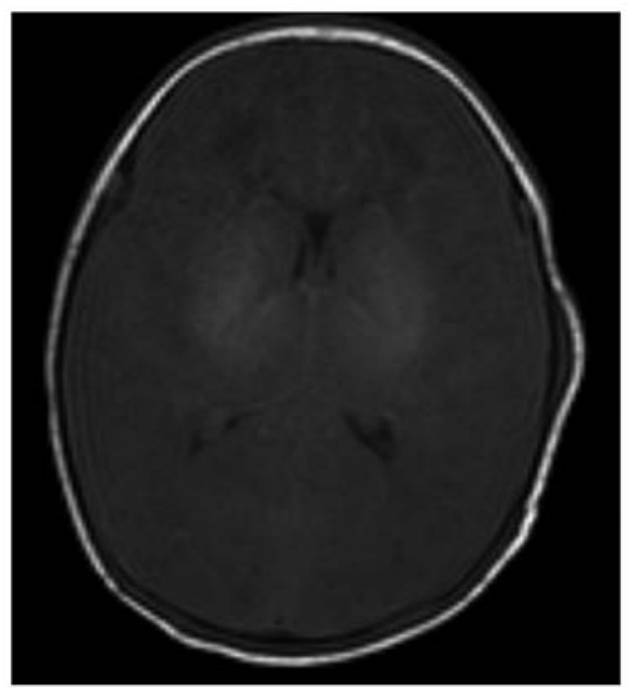

B

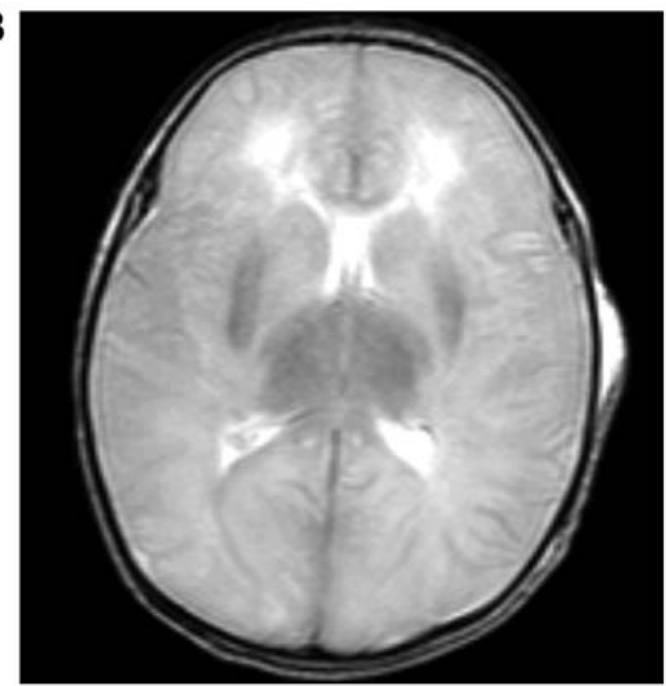

Figure 1. Magnetic resonance imaging of the head revealed diffuse low signal intensity in T1W1 and high signal intensity in T2W1 in comparison with a healthy patient and a marginal haziness in the cerebral gray matter of the cerebral hemisphere. (A) A slightly T1 weighted image can be seen in the gray and white matter of bilateral cerebral hemisphere, and (B) a slightly T2 weighted image can be seen in the gray and white matter of bilateral cerebral hemisphere without malformation of sulcus.

the citrulline level was $3.687 \mu \mathrm{M}$ (normal range, 4-30 $\mu \mathrm{M}$ ) and that glutamate level was $438.905 \mu \mathrm{M}$ (normal range, 100-400 $\mu \mathrm{M}$ ). The electrocardiogram (ECG) result was consistent with a normal neonatal ECG. Fasting blood sugar level was $4.5 \mathrm{mmol} / \mathrm{l}$ (normal range, 2.6-7.0 $\mathrm{mmol} / \mathrm{l}$ ). MRI of the cerebral hemisphere revealed diffuse low signal intensity in T1W1 and high signal intensity in T2W1 (compared with normal neonate) in the cerebral gray matter (Fig. 1).

Treatment. Head computed tomography excluded intracranial hemorrhage and the patient was suspected to have an intracranial infection. Thus, meropenem $(20 \mathrm{mg} / \mathrm{kg}$ once every $8 \mathrm{~h})$ combined with vancomycin (10 $\mathrm{mg} / \mathrm{kg}$ once every $12 \mathrm{~h}$ ) was administered to treat the infection, while g-globulin $(2.5 \mathrm{~g}$ in total), mannitol $(0.5 \mathrm{~g} / \mathrm{kg}$ once every $6 \mathrm{~h})$, furosemide $(0.5 \mathrm{~g} / \mathrm{kg}$ once every $12 \mathrm{~h}$ ), and albumin dehydration ( $1 \mathrm{~g} / \mathrm{kg}$ ) were used to reduce increased intracranial pressure and maintain electrolyte stability. On the 2nd day following admission, increased heart rate, decreased blood pressure, and mottled skin were noted and the symptoms relieved following active anti-shock treatment. The neonate lapsed into a deep coma, with a bulging fontanelle and increased tension. Upon receiving the results of routine blood analysis, on the 3rd day after admission, tests for the levels of CRP and procalcitonin were performed. The results of these tests did not support a bacterial infection. As cerebrospinal fluid may have no noticeable change from normal at an early stage of infection, the presence of a virus-induced intracranial infection could not be ruled out. The neonate was subjected to lumbar puncture manometry on the 3rd day following admission. Cerebrospinal fluid routine biochemical and virus examinations showed no evidence of intracranial infection. Blood ammonia levels were markedly increased (485.2 $\mu \mathrm{mol} / 1$; normal, $\leq 150 \mu \mathrm{mol} / \mathrm{l})$ while blood urea nitrogen $(\mathrm{BUN})$ was relatively low $(1.7 \mathrm{mmol} / \mathrm{l}$; normal, $2.8 \mathrm{mmol} / \mathrm{l}$ ). In consequence, the patient was diagnosed with encephalopathy caused by hyperammonemia. Accordingly, albumin was discontinued, and ammonia-lowering treatment, including high sugar, arginine, and body fluid supplements, was applied. Blood ammonia was almost normal $(75.5 \mu \mathrm{mol} / \mathrm{l})$ on the 5th day following admission, and remained in the normal range during the subsequent tests. However, the patient was in a state of deep coma, and liver size was markedly increased. Plasma amino acid examination on the 5th day following admission showed reduced citrulline levels and increased glutamate levels in comparison with a normal neonate, supporting the possibility of urea metabolism abnormality. On the 8th day following hospitalization, as the condition did not improve, the family discontinued treatment and the patient succumbed to the disease.

Compound heterozygosity in CPS1 is a potential candidate pathogenic variant. In order to reveal the genetic causes of the condition experienced by the patient, WES was performed. Upon filtration, compound heterozygous variants of c.713G $>C$, p.Arg238Pro and c.2339G >A, p.Arg780His in CPS1 (NCBI reference sequence, NM_001875.4) were regarded as candidate pathogenic mutations. The American College of Medical Genetics and Genomics hazard rating (21) for these variants was PM2 + PM3 + PP3 + PP1, which met the standard of 'likely pathogenic'. The c.2339G >A, p.Arg780His mutation was reported to be pathogenic (22). The p.Arg238Pro variant was expected to 'affect protein function' by SIFT software with a score of 0.00 , while it was predicted to be 'probably damaging' by PolyPhen 2 with a score of 0.976 (using the HumVar-trained prediction model), 'deleterious' by PROVEAN with a score of -6.33 and 'disease causing' by MutationTaster software with a score of 0.996 . The allele frequency of $\mathrm{c} .713 \mathrm{G}>\mathrm{C}$ was explored in four different single nucleotide polymorphism databases (SIFT, Polyphen2 HVAR, PROVEAN and MutationTaster). The results suggested that this novel mutation had not been previously registered (Table I). To validate the variants, Sanger sequencing of the family genetic profile was undertaken. The results illustrated that the mother was heterozygous for c.713G $>$ C, p.Arg238Pro and the father was heterozygous for 
c.2339G >A, p.Arg780His in CPS1, whereas the proband was heterozygous at both sites (Fig. 2).

Structural modeling demonstrates that the variants may influence the function of CPSI. To further assess the possible pathogenicity of these variants, sequence alignment together with structural modeling were undertaken. The whole sequence, including the identified sites of CPS1, is evolutionarily conserved across the species analyzed (Fig. 3A and B). In general, proteins with a highly conserved amino acid sequence are considered to be sensitive to variants, and hence, the identified sites were likely to be responsible for the etiology of the patient's condition (23). Based on structural data available online (http://www.rcsb.org/pdb/gene/) the apo form of the crystal structure of CPS1 was also studied. As depicted in Fig. 3C, R238 is located at the terminal region of an $\alpha$-helix and forms hydrogen bonds with D392, which is located on an adjacent $\alpha$-helix. In addition, R238 forms hydrogen bonds with L234. These interactions serve crucial roles in the local structure of these two helixes. The R238P variant changes the charge characteristic of the side chain and may disrupt the local structure. In light of this, it is possible that the R238P variant may affect the function of CPS1. The R780H variant maps in a loop key for the transmission of the allosteric signal (20), and this mutation has been previously reported to be pathogenic (22). Accordingly, the variants identified in the proband may cause CPS1 deficiency.

\section{Discussion}

The current study presented the clinical performance, diagnosis, and treatment of CPS1 deficiency in a neonate. At the time of admission, the patient exhibited a mild coma, persistent fever, high fontanelle tension, high muscle tension and repeated convulsions. Additionally, the patient temperature at admission was $\sim 40.2^{\circ} \mathrm{C}$. As a result, the following diseases were initially suspected: Neonatal encephalopathy, intracranial infection, intracranial hemorrhage, septicemia or hereditary metabolic disease. The patient was finally diagnosed with $\mathrm{UCD}$, due to the symptoms of encephalopathy and hyperammonemia. Abnormal blood ammonia in comparison with the level in a normal child was not noted at the time of admission. However, ammonia level had significantly increased by the $3^{\text {rd }}$ day following admission when compared with the level in a normal child. A possible cause for this may be that no milk intake occurred within $20 \mathrm{~h}$; thus, there was no protein substrate intake, masking the potential diagnosis (9). Upon admission, although amino acids were not supplemented, albumin dehydration and craniofacial pressure treatment were administered. In addition, protein substrate was added and the blood ammonia increased significantly. In line with previously published guidelines, protein intake should be avoided before UCD has been excluded (24).

CPS1 deficiency presenting as anorexia, irregular breathing, fever, convulsions, poor mental response, and coma occurred on the 3 rd day after birth. The symptoms were similar to the presentation of a newborn reported by Yang et al (25). CPS1 deficiency can easily be misdiagnosed as septicemia and intracranial infection (26). The findings of the present study suggest that CPS1 deficiency should be considered when 


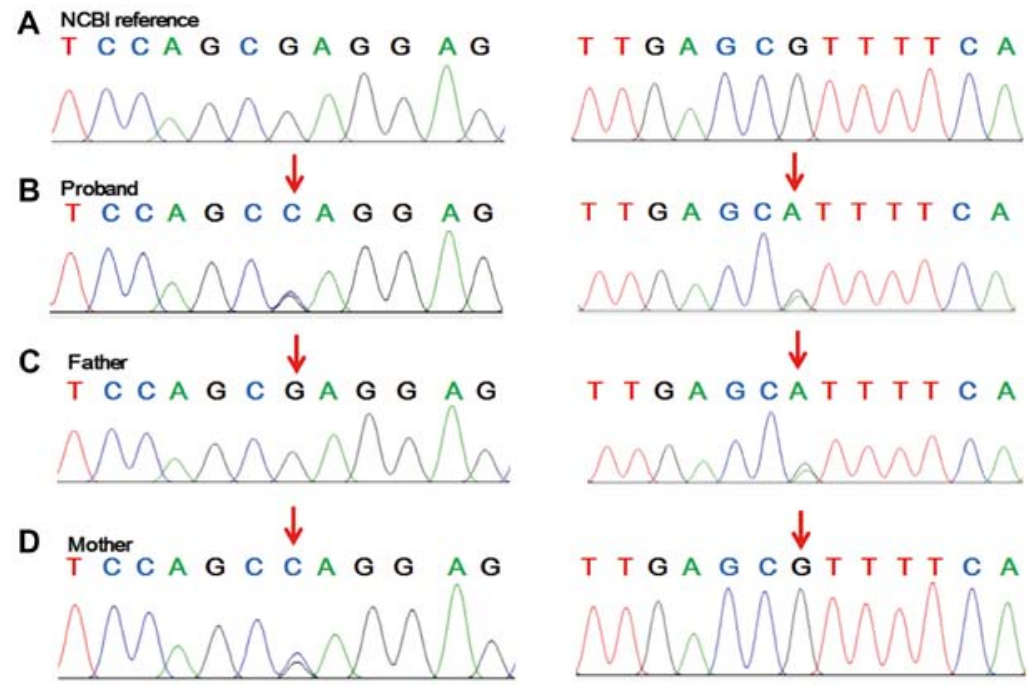

Figure 2. Sanger sequencing of the three family members revealed that the patient had heterozygous variants of (left) c.713G $>C$ (p.Arg238Pro) and (right) c.2339G $>$ A (p.Arg780His) in the carbamoyl phosphate synthetase 1 gene (NCBI reference sequence, NM_001875.4), which were inherited from the parents. (A) NCBI reference sequence. Sequences obtained from (B) the proband, (C) father, and (D) mother. NCBI, National Center for Biotechnology Information.

A

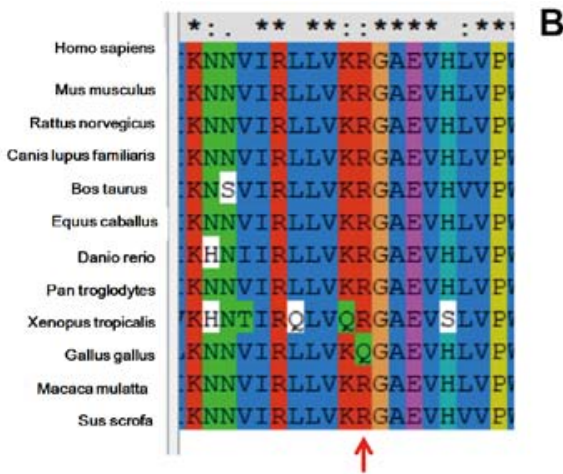

B

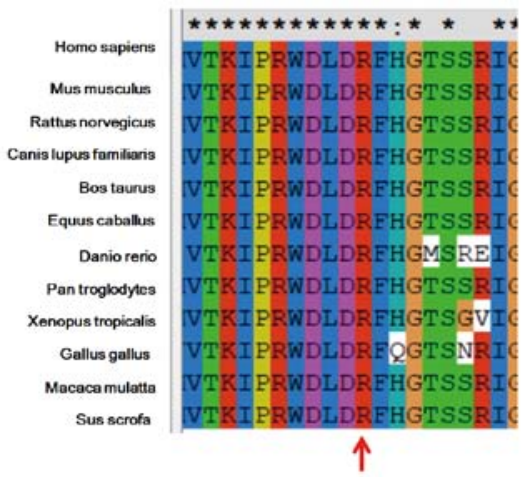

\section{C}

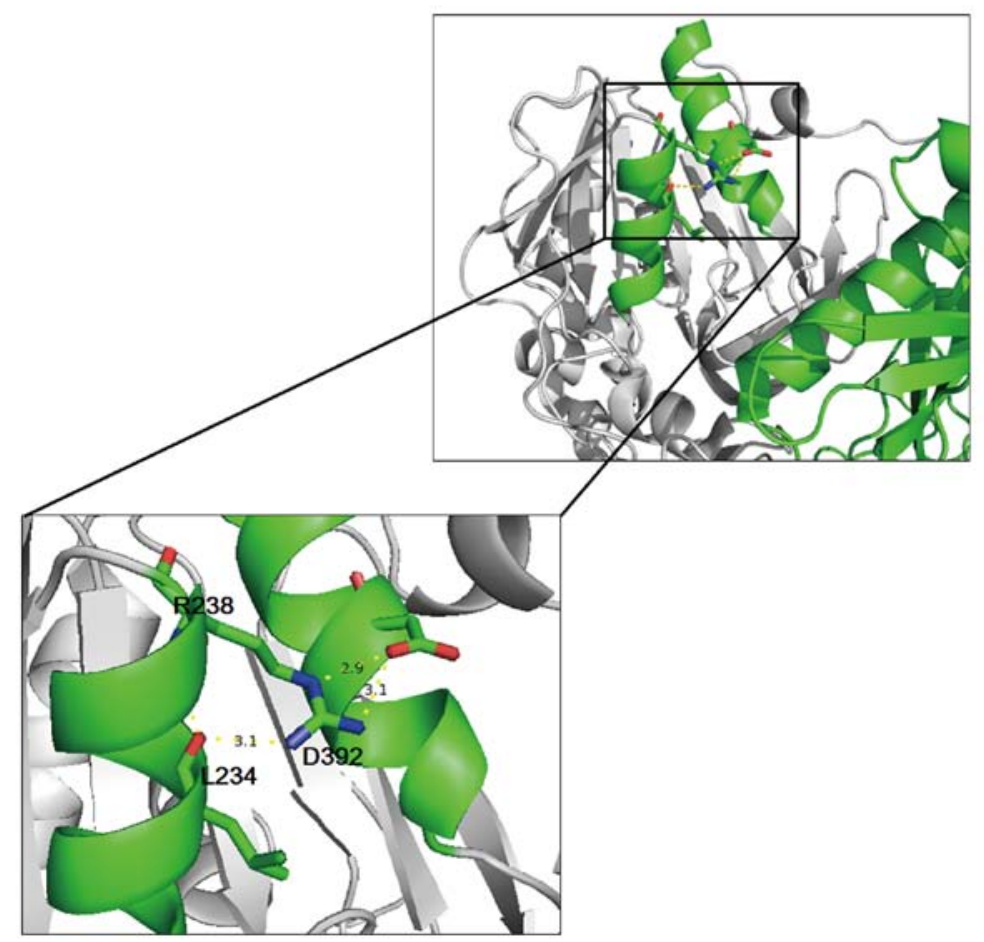

Figure 3. Sequence alignment and structural modeling results for the variants. Sequence alignment around the variant sites of (A) c.713G $>C$ (p.Arg238Pro, red arrow) and (B) c.2339G>A (p.Arg780His, red arrow) showed that CPS1 was conserved among different species. The symbols '*', '?' and ' ' indicate a high level of sequence conservation, where *>:>. (C) Structural modeling demonstrated that the c.713G $>C$ (p.Arg238Pro) variant may disrupt the local structure of CPS1. CPS1, carbamoyl phosphate synthetase 1 . 
intracranial hemorrhage, hypoglycemia, and electrolyte disorders are suspected. For children with unexplained vomiting, anorexia, poor mental reaction and convulsions, repeated blood ammonia monitoring should be performed. Citrulline and orotic acid levels are helpful in identifying the subtypes of UCD (27). Children with CPS1 deficiency typically exhibit plasma glutamic acid increase, citrulline decrease, and normal urinary orotic acid levels (28). Genetic testing such as WES can further validate a diagnosis and provide a reliable basis for prenatal eugenics (12). However, WES is a lengthy process from test to the final diagnosis, and therefore, it should be performed as soon as possible in patients who are difficult to give diagnose clinically. The patient in the present study was identified to have compound heterozygous variants of c.713G $>C$, Arg 238Pro and c. $2339 \mathrm{G}>\mathrm{A}$, Arg780His in the CPS1 gene. The c.2339G $>\mathrm{A}, \mathrm{Arg} 780 \mathrm{His}$ variant is a known pathogenic mutation (22) while c.713G $>\mathrm{C}, \operatorname{Arg} 238$ Pro is, to the best of our knowledge, a novel heterozygous mutation not included in any database. CPS1 c.2339G $>$ A has been included in the Genome Aggregation Database (gnomAD) and the Exome Aggregation Consortium (ExAC) with very rare frequencies $\left(1.629 \times 10^{-05}\right.$ and $3.304 \times 10^{-05}$, respectively). This variant has been included in the ClinVar and HGMD databases and could be classified as likely pathogenic. CPS1 c.713G>C, p.Arg238Pro has not been reported in gnomAD, ExAC, 1000G or HGMD. However, for this amino acid site, p.R238Ter has been included in HGMD. Therefore, the clinical interpretation of this variant could be classified as likely pathogenic. Protein functional prediction and structural modeling suggested that the mutation was harmful. In line with this, it is possible that the proband exhibited CPS1 deficiency. Thus, the present report focused on the genetic spectrum of this disease.

Treatment for patients with CPS1 deficiency and clinical symptoms of hyperammonia includes limiting protein intake, using drugs to reduce serum ammonia levels (L-arginine, sodium benzoate, and sodium phenylacetate), and supplementing with glucose (20). For severe ammonia levels, blood filtration should be considered (24). The patient in the present study had early onset disease with rapid progress and a poor prognosis. If limited protein intake prior to coma had been undertaken, excessive blood ammonia may have been avoided and the prognosis may have been improved. Reports indicate that the onset of CPS1 deficiency in the neonatal period is often fatal, and the severity of clinical manifestations depends on the time of onset and the level of residual enzymatic activity $(29,30)$. Certain patients who receive long-term protein restriction and ammonia removal therapy may also have sequelae (31). A previous study reported a patient who had remission following liver transplantation (30).

Collectively, hereditary metabolic diseases in the neonatal period often lead patients to deteriorate rapidly. Therefore, early recognition of the disease by clinicians is of great importance (13). The findings of the present study suggest that blood ammonia monitoring should be carried out in cases where seemingly healthy newborns display uncommon symptoms several days after milk intake, in order to exclude UCD. This monitoring may facilitate early diagnosis and treatment and improve the quality of life of these patients.

\section{Acknowledgements}

Not applicable.

\section{Funding}

This study was supported by Changsha Science and Technology Bureau Project (grant no. KQ1602027).

\section{Availability of data and materials}

The datasets used and/or analyzed during the present study are available from the corresponding author on reasonable request.

\section{Authors' contributions}

JX and AZ designed the study. JX collected the clinical data, performed the experiments and drafted the manuscript. FH participated in clinical data collection. All authors read and approved the final version of the manuscript.

\section{Ethics approval and consent to participate}

The present study was approved by the Ethics Committee of Hunan Provincial People's Hospital (Changsha, China) and informed consent was obtained from parents of the proband.

\section{Patient consent for publication}

Consent for publication was obtained from the parents of the proband.

\section{Competing interests}

All authors declare that they have no competing interests.

\section{References}

1. Soria LR, Allegri G, Melck D, Pastore N, Annunziata P, Paris D, Polishchuk E, Nusco E, Thöny B, Motta A, et al: Enhancement of hepatic autophagy increases ureagenesis and protects against hyperammonemia. Proc Natl Acad Sci U S A 115: 391-396, 2018.

2. Ballantyne LL, Sin YY, St Amand T, Si J, Goossens S, Haenebalcke L, Haigh JJ, Kyriakopoulou L, Schulze A and Funk CD: Strategies to rescue the consequences of inducible arginase-1 deficiency in mice. PLoS One 10: e0125967, 2015.

3. Braissant O: Current concepts in the pathogenesis of urea cycle disorders. Mol Genet Metab 100 (Suppl 1): S3-S12, 2010.

4. Unsinn C, Das A, Valayannopoulos V, Thimm E, Beblo S, Burlina A, Konstantopoulou V, Mayorandan S, de Lonlay P, Rennecke J, et al: Clinical course of 63 patients with neonatal onset urea cycle disorders in the years 2001-2013. Orphanet J Rare Dis 11: 116, 2016.

5. Summar ML, Dasouki MJ, Schofield PJ, Krishnamani MR, Vnencak-Jones C, Tuchman M, Mao J and Phillips JA 3rd: Physical and linkage mapping of human carbamyl phosphate synthetase I (CPS1) and reassignment from $2 p$ to $2 q 35$. Cytogenet Cell Genet 71: 266-267, 1995.

6. Haberle J, Schmidt E, Pauli S, Rapp B, Christensen E, Wermuth B and Koch HG: Gene structure of human carbamylphosphate synthetase 1 and novel mutations in patients with neonatal onset. Hum Mutat 21: 444, 2003.

7. Funghini S, Donati MA, Pasquini E, Zammarchi E and Morrone A: Structural organization of the human carbamyl phosphate synthetase I gene (CPS1) and identification of two novel genetic lesions. Hum Mutat 22: 340-341, 2003. 
8. Summar ML, Hall LD, Eeds AM, Hutcheson HB, Kuo AN, Willis AS, Rubio V, Arvin MK, Schofield JP and Dawson EP: Characterization of genomic structure and polymorphisms in the human carbamyl phosphate synthetase I gene. Gene 311: 51-57, 2003.

9. Singh RH: Nutritional management of patients with urea cycle disorders. J Inherit Metab Dis 30: 880-887, 2007.

10. World Medical Association: World medical association declaration of helsinki: Ethical principles for medical research involving human subjects. JAMA 310: 2191-2194, 2013.

11. Jin D, Yu T, Zhang L, Wang T, Hu J, Wang Y and Yang XA: Novel NFU1 variants induced MMDS behaved as special leukodystrophy in chinese sufferers. J Mol Neurosci 62: 255-261, 2017.

12. Peng F, Zhong L, Zhang B, Zou R, Nie S, Tian X, Deng S and $\mathrm{He}$ X: Successful application of next-generation sequencing for pre-natal diagnosis in a pedigree with chronic granulomatosis disease. Exp Ther Med 17: 2931-2936, 2019.

13. Chen Q, Bao H, Wu H, Zhao S, Huang S and Zhao F: Diagnosis of cobalamin $\mathrm{C}$ deficiency with renal abnormality from onset in a Chinese child by next generation sequencing: A case report. Exp Ther Med 14: 3637-3643, 2017.

14. Zytkovicz TH, Fitzgerald EF, Marsden D, Larson CA, Shih VE, Johnson DM, Strauss AW, Comeau AM, Eaton RB and Grady GF: Tandem mass spectrometric analysis for amino, organic, and fatty acid disorders in newborn dried blood spots: A two-year summary from the new England newborn screening program. Clin Chem 47: 1945-1955, 2001.

15. Adzhubei I, Jordan DM and Sunyaev SR: Predicting functional effect of human missense mutations using PolyPhen-2. Curr Protoc Hum Genet 7: 20, 2013

16. Kumar P, Henikoff S and Ng PC: Predicting the effects of coding non-synonymous variants on protein function using the SIFT algorithm. Nat Protoc 4: 1073-1081, 2009.

17. Choi Y, Sims GE, Murphy S, Miller JR and Chan AP: Predicting the functional effect of amino acid substitutions and indels. PLOS One 7: e46688, 2012.

18. Choi Y and Chan AP: PROVEAN web server: A tool to predict the functional effect of amino acid substitutions and indels. Bioinformatics 31: 2745-2747, 2015.

19. Schwarz JM, Cooper DN, Schuelke M and Seelow D MutationTaster2: Mutation prediction for the deep-sequencing age. Nat Methods 11: 361-362, 2014.

20. de Cima S, Polo LM, Diez-Fernandez C, Martínez AI, Cervera J, Fita I and Rubio V: Structure of human carbamoyl phosphate synthetase: Deciphering the on/off switch of human ureagenesis. Sci Rep 5: 16950, 2015.
21. Richards S, Aziz N, Bale S, Bick D, Das S, Gastier-Foster J, Grody WW, Hegde M, Lyon E, Spector E, et al: Standards and guidelines for the interpretation of sequence variants: A joint consensus recommendation of the American college of medical genetics and genomics and the association for molecular pathology. Genet Med 17: 405-424, 2015.

22. Kurokawa K, Yorifuji T, Kawai M, Momoi T, Nagasaka H, Takayanagi M, Kobayashi K, Yoshino M, Kosho T, Adachi M, et al: Molecular and clinical analyses of Japanese patients with carbamoylphosphate synthetase 1 (CPS1) deficiency. J Hum Genet 2: 349-354, 2007.

23. Liu D, Wang Y, Yang XA and Liu D: De novo mutation of paternal IGF2 gene causing silver-russell syndrome in a sporadic patient. Front Genet 8: 105, 2017.

24. Haberle J, Boddaert N, Burlina A, Chakrapani A, Dixon M, Huemer M, Karall D, Martinelli D, Crespo PS, Santer R, et al: Suggested guidelines for the diagnosis and management of urea cycle disorders. Orphanet J Rare Dis 7: 32, 2012.

25. Yang X, Shi J, Lei H, Xia B and Mu D: Neonatal-onset carbamoyl phosphate synthetase I deficiency: A case report. Medicine (Baltimore) 96: e7365, 2017.

26. Ali EZ, Khalid MK, Yunus ZM, Yakob Y, Chin CB, Abd Latif K and Hock NL: Carbamoylphosphate synthetase 1 (CPS1) deficiency: Clinical, biochemical, and molecular characterization in Malaysian patients. Eur J Pediatr 175: 339-346, 2016.

27. Haberle J, Burlina A, Chakrapani A, Dixon M, Karall D, Lindner M, Mandel H, Martinelli D, Pintos-Morell G, Santer R, et al: Suggested guidelines for the diagnosis and management of urea cycle disorders: First revision. J Inherit Metab Dis 42: 1192-1230, 2019.

28. Ono H, Suto T, Kinoshita Y, Sakano T, Furue T and Ohta T: A case of carbamoyl phosphate synthetase 1 deficiency presenting symptoms at one month of age. Brain Dev 31: 779-781, 2009.

29. Klaus V, Vermeulen T, Minassian B, Israelian N, Engel K, Lund AM, Roebrock K, Christensen E and Häberle J: Highly variable clinical phenotype of carbamylphosphate synthetase 1 deficiency in one family: An effect of allelic variation in gene expression? Clin Genet 76: 263-269, 2009.

30. Foschi FG, Morelli MC, Savini S, Dall'Aglio AC,Lanzi A, Cescon M, Ercolani G, Cucchetti A, Pinna AD and Stefanini GF: Urea cycle disorders: A case report of a successful treatment with liver transplant and a literature review. World J Gastroenterol 21: 4063-4068, 2015.

31. Kasahara M, Sakamoto S, Shigeta T, Fukuda A, Kosaki R, Nakazawa A, Uemoto S, Noda M, Naiki Y and Horikawa R: Living-donor liver transplantation for carbamoyl phosphate synthetase 1 deficiency. Pediatr Transplant 14: 1036-1040, 2010. 